Cite this: J. Mater. Chem. B, 2013, 1, 571

Received 12th October 2012 Accepted 14th November 2012 DOI: $10.1039 / c 2$ tb00248e

www.rsc.org/MaterialsB

\title{
Polyurethane (PU)-derived photoactive and copper-free clickable surface based on perfluorophenyl azide (PFPA) chemistry†
}

\author{
Lingdong Li, Jiang Li, Abhilash Kulkarni and Song Liu*
}

The anchoring and capturing roles of perfluorophenyl azide (PFPA) were combined to produce a universal polyurethane (PU)-derived photoactive surface platform (PU-1-PFPA). The resultant platform was confirmed by contact angle, attenuated total reflectance Fourier transform infrared (ATR FT-IR) spectroscopy, atomic force microscopy (AFM) and X-ray photoelectron spectroscopy (XPS) analyses. Upon UV light activation, native heparin was coupled directly onto PU-1-PFPA to yield a substrate with antithrombogenic properties. The same level of antithrombogenic activity was achieved when the recovered heparin was photo-coupled onto PU-1-PFPA. In addition, at room temperature and in the absence of copper catalysts, PU-1-PFPA achieved oriented immobilization of functional moieties bearing an alkynyl functional group.

\section{Introduction}

Synthetic polymeric materials such as poly(ethylene terephthalate) (PET), polyurethane (PU), and poly(lactic acid) (PLA) have been widely used for medical applications including vascular grafts, ligament and tendon prostheses, and tissue engineering scaffolds. ${ }^{\mathbf{1} 2}$ However, often these materials do not have the appropriate surface properties essential for inciting in situ tissue regeneration ${ }^{3}$ or for avoiding adverse biological responses such as thrombosis and inflammatory response. ${ }^{4}$ Therefore, bioactive agents are usually required to be immobilized on material surfaces to help meet the biological challenges at the tissue-biomaterial interface.

On surfaces with functional groups such as hydroxyl and amine groups, many coupling agents can be easily introduced to serve as linker molecules for the eventual immobilization of the bioactive compounds. ${ }^{5-7}$ However, the choice of a coupling agent universally applicable to a wide range of polymeric substrates is relatively limited. The universal chemistry for covalent surface modification of polymeric substrates is generally based on the generation of reactive radicals (from Norrish II initiators), carbenes and nitrenes. Radicals generated from Norrish type II initiators have a high tendency to abstract hydrogen from substrate polymers. The subsequent coupling of the substrate

Department of Textile Sciences, Faculty of Human Ecology, Department of Chemistry, Faculty of Science, University of Manitoba, Winnipeg, Canada R3T 2N2. E-mail: Song. Liu@ad.umanitoba.ca; Fax: +1 204-474-7593; Tel: +1 204-474-9616

$\uparrow$ Electronic supplementary information (ESI) available: Synthesis attempt on compound 4 and characterization of PU-3 including ATR, AFM and calculation of surface functionalization level, and the self-click phenomenon on a TLC plate between PFPA and alkynes. See DOI: 10.1039/c2tb00248e radical and the primary radical generated from the initiator produces a covalent attachment of the initiator molecule. Benzophenone, a typical Norrish II initiator, has been successfully grafted onto poly(dimethylsiloxane), ${ }^{8}$ polyethylene, ${ }^{9}$ polypropylene, ${ }^{\mathbf{1 0}}$ and PET. $^{\mathbf{1 1}}$ If benzophenone is derivatized with functional groups, ${ }^{\mathbf{1 1}}$ direct functionalization of the substrate polymer could be achieved. Upon exposure to UV irradiation (365 nm), the benzophenone moiety anchors to the surface of bulk polymers so as to introduce new functional groups. Photogenerated carbenes and nitrenes preferably insert into $\mathrm{C}-\mathrm{H}, \mathrm{N}-\mathrm{H}$ and $\mathrm{C}=\mathrm{C}$ to form covalent bonds. Two typical corresponding photoreagents are trifluoromethyl-aryldiazirine ${ }^{\mathbf{1 2}}$ and perfluorophenyl azide (PFPA) ${ }^{\mathbf{1 3}}$ and these two photoreagents can also be derivatized with to-be-immobilized target functional groups.

After the introduction of functional groups onto inert polymeric substrates using photoimmobilization strategies, secondary chemistry is needed for the covalent bonding of biomolecules. For example, aldehyde groups were introduced onto the surface of nylon membranes by photoactivating $p$-azidobenzaldehyde coated on the membrane, and subsequently various antibodies were covalently bound through a reaction with the surface aldehyde groups. ${ }^{\mathbf{1 4}}$ The photosensitive heads of the three photoreagents, i.e. benzophenone, trifluoromethyl aryldiazirine and perfluorophenyl azide (PFPA), can also covalently capture biomolecules onto the surfaces if these photoreagents are first immobilized via thermochemical reactions. For instance, PFPA has been immobilized on silicon oxide $^{15}$ and metal nanoparticles ${ }^{16,17}$ using silane chemistry and transition metal sulfur chemistry respectively, resulting in photoactive surfaces for secondary functionalization.

The combination of photo-anchoring and photo-capturing has attracted interest from investigators for some time. Sigrist 
and coworkers ${ }^{\mathbf{1 8}}$ reported a one-step photoimmobilization of biomolecules (streptavidin, inulin, and encephalin) onto polystyrene in the presence of trifluoromethyl aryldiazirine derivatized bovine serum albumin (BSA) whose degree of photolabeling was 8-10 mol trifluoromethyl aryldiazirine per mol BSA. Upon light activation, some of the generated carbenes along the backbone of BSA insert into the polystyrene substrate while the others insert into the biomolecules, producing a covalent grafting of the biomolecules onto the chemically inert polystyrene. Pei et al. ${ }^{19}$ realized a carbohydrate microarray through multi-steps of photo-capturing and photo-anchoring: following the photo-capturing of poly(ethylene oxide) onto PFPA functionalized glass slides, PFPA derivatized carbohydrates were photo-anchored onto the surface in an arrayed manner. Although there is a trend to incorporate both photo-anchoring and photo-capturing processes in surface ligation, ${ }^{\mathbf{1 8 , 1 9}}$ the combination of PFPA photo-anchoring/capturing strategy to covalently immobilize functional moieties onto thermoplastic polymers lacking reactive groups has not been explored.

Photoimmobilization strategies are extremely useful to introduce reactive functions on chemically inert surfaces and to capture biomolecules lacking reactive functional groups. However, the abovementioned photoimmobilization chemistry suffers from one problem: it lacks functional group specificity. Nevertheless, the photoreagent PFPA carries an azide functional group which has been reported to react orthogonally and quantitatively with an alkyl group under the catalysis of cop$\operatorname{per}(\mathrm{I}) .{ }^{20}$ So, we hypothesize that we can build a versatile and flexible surface platform for both the immobilization of bioactive agents lacking reaction groups and the oriented immobilization of alkynyl functional moieties by combining the photoreactivity and click potential of PFPA.

PU has been used extensively in biomedical applications ${ }^{\mathbf{2 1 , 2 2}}$ due to its elastic and compliant mechanical properties. However, its hemocompatibility is inadequate, especially for use in small diameter blood vessels $(\leq 6 \mathrm{~mm})$. To improve its hemocompatibility, a variety of materials such as poly(ethylene glycol) (PEG), ${ }^{23}$ phospholipid polymers, ${ }^{24}$ sulfobetain, ${ }^{25}$ and heparin ${ }^{26}$ have been integrated onto the PU surface. The most widely used anticoagulant, heparin, has been introduced onto the polymer surface via either physisorption or covalent bonding. The covalent bonding is usually achieved by treating amine furnished polymeric substrates with 1-ethyl-3-(3-dimethylaminopropyl)carbodiimide/ $N$-hydroxysuccinimide (EDC/ NHS) activated heparin. However, since EDC/NHS is reportedly cytotoxic, extensive after-modification rinsing of the resultant materials is necessary. ${ }^{27}$ On the other hand, it was reported that carboxyl groups grafted onto a PU film could effectively prolong the partial thromboplastin time (APTT). ${ }^{28}$ Therefore, if heparin is immobilized at the expense of some carboxyl groups, the ratio of carboxyl group/sulfonic group decreases, probably contributing to the compromise of heparin bioactivity after its immobilization. Thus far, the development of simple and effective heparin immobilization methods via covalent linkage has remained an important goal.

Based on the concept of PFPA chemistry, ${ }^{13}$ we proposed to use light activation to introduce PFPA onto PU for the purpose of integrating surface functionalities. Yan and coworkers have successfully photoanchored 2,5-dioxopyrrolidin-1-yl-4-azido2,3,5,6-tetrafluorobenzoate (PFPA-NHS (1)) on polystyrene (PS) ${ }^{29}$ to give the surface an NHS-platform, onto which amine containing biomolecules can be coupled. Proteins and oligonucleotides have been covalently attached onto the NHS-platform for biological sensing and imaging applications, ${ }^{30,31}$ but this methodology does not work in the case of amine-free target bioactive reagents such as heparin. We proposed to confer PFPA-amine (2) onto the NHS-platform to afford a surface photoactive platform (SPP) onto which underivatized functional cargoes of interest can be photo-coupled.

In this work, we photo-coupled 1 onto a PU film (PU-1) and subsequently attached 2 onto PU-1 to fabricate the general SPP as PU-1-PFPA (Scheme 1). The modified surface was characterized using contact angle measurements, attenuated total reflectance Fourier transform infrared (ATR FT-IR) spectroscopy, atomic force microscopy (AFM) and X-ray photoelectron spectroscopy (XPS). Without chemical derivatization, heparin was successfully photo-immobilized onto PU-1-PFPA using UV irradiation. Platelet adhesion to the resultant PU film was proven to be significantly less than that to the untreated PU film. The constructed platform (PU-1-PFPA) can not only capture heparin photochemically, but can also conjugate with alkynyl functional moieties in the absence of toxic copper catalysts.

\section{Experimental section}

\section{Materials and agents}

The solvents and chemicals such as methyl pentafluorobenzoate, 2-propynylamine, 2,2-methylbut-3-yn-1-amine, 4-chlorobutanoyl chloride, dansyl chloride ethylenediamine, propargyl alcohol, hex-5-yn-1-ol, 1-ethyl-3-(3-dimethylaminopropyl)carbodiimide (EDC), $N$-hydroxysuccinimide (NHS), heparin (sodium salt), toluidine blue and Orange Acid II were purchased from Aldrich or VWR and used without further purification unless otherwise noted. The silica gel was received from Selecto Scientific, Georgia, USA, while the TLC plate was from Analtech, Inc., USA. The PU film (gauge: 6 mil) was purchased from American Polyfilm, Inc.

According to known procedures, ${ }^{32,33}$ perfluorophenyl azide and its derivatives were prepared as depicted in Scheme 2. In brief, substitution of the commercial methyl pentafluorobenzoate with sodium azide was completed under acetone reflux conditions to give the $p$-azido counterpart $4 .^{32}$ Subsequent hydrolysis under alkaline conditions $(\mathrm{NaOH})$ afforded the $p$-azidotetrafluorobenzoic acid $3 .^{32}$ Then the corresponding active ester 1 was prepared by coupling 3 with NHS, and subsequently the PFPA-amine 2 was obtained by treating 1 with excess ethylenediamine. ${ }^{33}$ Purification of the synthetic compounds was performed by flash column chromatography. All obtained NMR data were identical to those found in the literature. Azido-compounds, including 2-azido-ethylamine and dansyl-azide 5 (Scheme 2), were prepared according to a published protocol. ${ }^{34,35}$

SYNTHESIS OF DANSYL-ALKYNE (6). Compound 6 was prepared using a method similar to the synthesis of $5 .^{35}$ To dansyl 

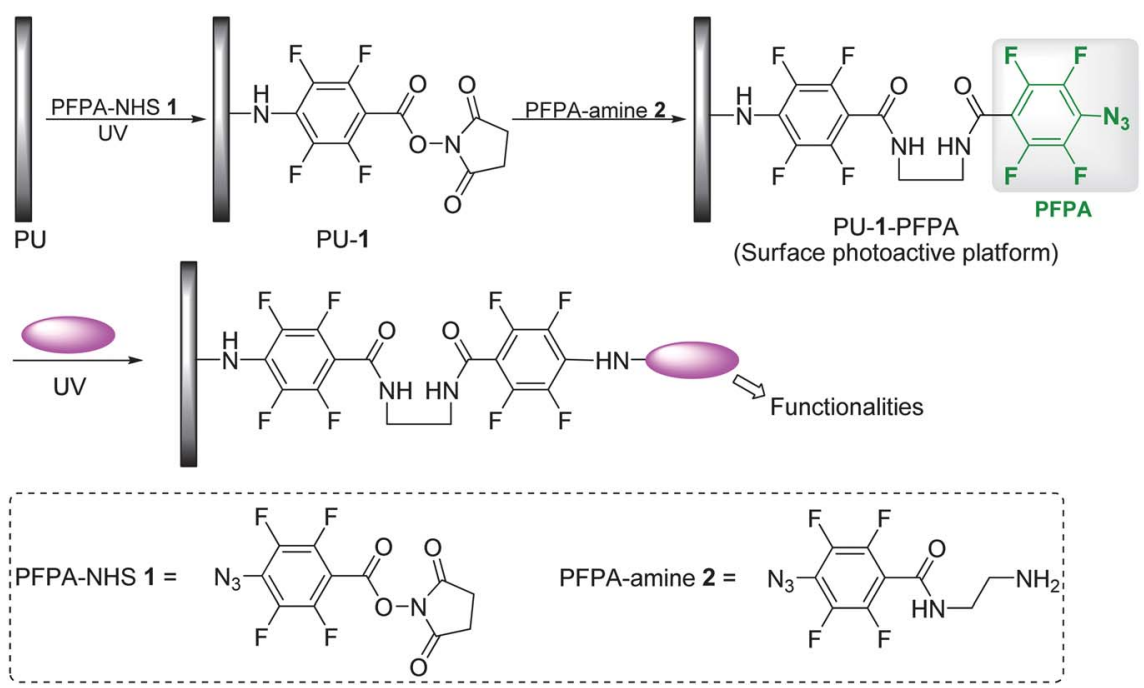

Scheme 1 Schematic illustration of the PU-derived surface photoactive platform (SPP).
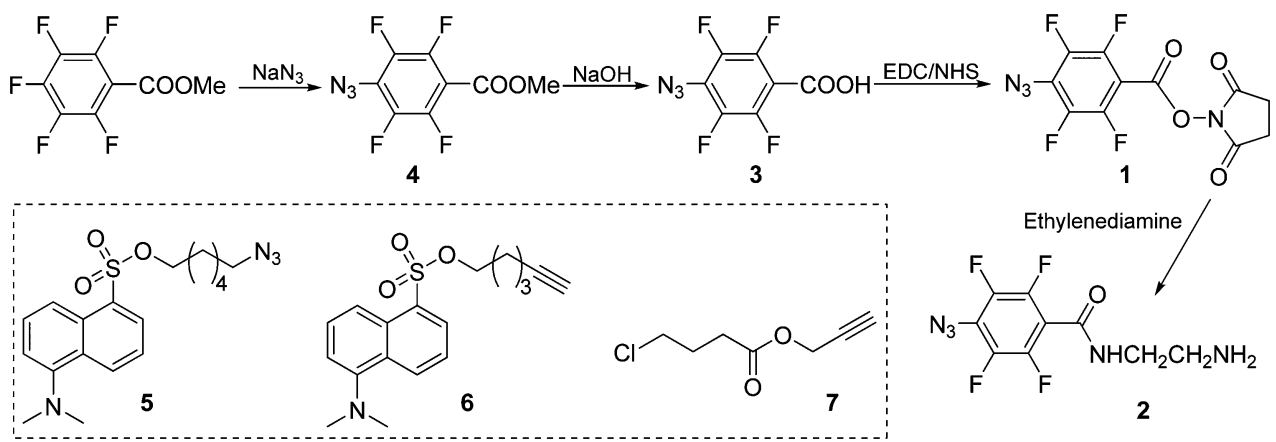

Scheme 2 Synthesis of PFPA derivatives and major compounds used in this work.

chloride $(1.04 \mathrm{~g}, 3.8 \mathrm{mmol})$ solution in $\mathrm{CH}_{2} \mathrm{Cl}_{2}(12 \mathrm{~mL})$ and 0.5 $\mathrm{mL}$ pyridine, excess hex-5-yn-1-ol $(0.39 \mathrm{~g}, 4.0 \mathrm{~mol})$ was added. The resulting solution was stirred for $24 \mathrm{~h}$ before being concentrated under vacuum. The residue was loaded onto a silica gel column and washed with EtOAc-Hex $(1: 8)$. The fluorescent fractions were combined to afford 6 as fluorescent oil (1.09 g, 86\%); ${ }^{1} \mathrm{H}$ NMR $\left(\mathrm{CDCl}_{3}, 300 \mathrm{MHz}\right) \delta 8.69(\mathrm{~d}, J=8.1 \mathrm{~Hz}$, $1 \mathrm{H}), 8.29-8.34(\mathrm{~m}, 2 \mathrm{H}), 7.56-7.65(\mathrm{~m}, 2 \mathrm{H}), 7.26-7.28(\mathrm{~m}, 1 \mathrm{H})$, $4.04(\mathrm{t}, J=6.2 \mathrm{~Hz}, 2 \mathrm{H}), 2.95(\mathrm{~s}, 6 \mathrm{H}), 2.06-2.11(\mathrm{~m}, 2 \mathrm{H}), 1.89(\mathrm{t}$, $J=2.6 \mathrm{~Hz}, 1 \mathrm{H}), 1.71-1.80(\mathrm{~m}, 2 \mathrm{H}), 1.47-1.56(\mathrm{~m}, 2 \mathrm{H}) ;{ }^{13} \mathrm{C} \mathrm{NMR}$ $\left(\mathrm{CDCl}_{3}, 75 \mathrm{MHz}\right) \delta 167.8,150.8,131.5,131.3,130.9,130.5,129.6$, 123.4, 120.1, 115.9, 83.3, 70.3, 68.9, 45.6, 27.7, 24.2, 17.6.

SYNTHESIS OF PROP-2-YNYL 4-CHLOROBUTANOATE (7). To the solution of propargyl alcohol $(1.0 \mathrm{~g}, 17.8 \mathrm{mmol}$, in $15 \mathrm{~mL}$ dichloromethane), triethylamine $(1.36 \mathrm{~mL}, 9.8 \mathrm{mmol})$ was added at $0{ }^{\circ} \mathrm{C}$. Subsequently, 4-chlorobutanoyl chloride $(1.26 \mathrm{~g}$, $8.9 \mathrm{mmol}$ ) was added dropwise over 10 minutes while stirring. After stirring at ambient temperature overnight, the solution was filtered and the solvent removed under reduced pressure. The obtained residue was purified by column chromatography to give a slightly yellow liquid $\left(1.2 \mathrm{~g} 85 \%,{ }^{1} \mathrm{H}\right.$ NMR data were identical to commercial products from Aurora Fine Chemicals LLC, USA).

\section{Instrumental analysis and characterization}

ATR FT-IR spectra were taken on a Nicolet is10 spectrometer (Thermo Electron Corporation). NMR spectra were recorded at room temperature (RT) in $5 \mathrm{~mm}$ NMR tubes on a Bruker Avance $300 \mathrm{MHz}$ NMR spectrometer. XPS data were acquired with a Kratos Axis Ultra spectrometer from Kratos Analytical, Inc. The contact angle was measured by a standard contact angle goniometer (Model 200, with DROPimage standard) from RaméHart Inc. AFM spectra were obtained using a nanoscope multimedia IIIa atomic force microscope from Veeco Instruments Inc. The images were taken under a tapping mode.

\section{General procedure for PU surface photo-functionalization}

Methanol solution of 1 or 3 (4.0 $\mathrm{mg} \mathrm{mL}^{-1}$, Scheme 2) was cast on the PU film and the resulting film was baked for $20 \mathrm{~min}$ in an oven preheated to $60{ }^{\circ} \mathrm{C}$. Then the film was exposed to UV irradiation (254 nm lamp, $1690 \mu \mathrm{W} \mathrm{cm}{ }^{-2}$ ) for $10 \mathrm{~min}$ at a distance of $5 \mathrm{~cm}$. After a thorough rinse with methanol, the film was air dried and stored at $4{ }^{\circ} \mathrm{C}$ before use (denoted as PU-1 and PU-3 respectively).

The same process was repeated to produce PU-3/PU-1 samples for dye staining. A drop of 3/1 methanol solution was 
spotted on the PU film; after photolysis, the film sample was rinsed with ethanol to give samples PU-3-s and PU-1-s respectively ( $\mathrm{s}=$ spotting). PU-3-s was then immersed in $0.1 \mathrm{mg} \mathrm{mL}$ thionin acetate (THA) solution for $8 \mathrm{~h}$, excess dye was washed off with ethanol, and an image was taken.

\section{Titration of the surface carboxylic acid group on PU-3}

The surface carboxylic acid group on PU-3 was determined by monitoring the absorbance change of THA dye solution before and after the electrostatic interaction driven dyeing process using a known procedure. ${ }^{36}$ The PU-3 films were immersed in $5 \mathrm{~mL}$ of a THA solution in ethanol (concentration $0.1 \mathrm{mg} \mathrm{mL}^{-1}$ ) and the mixture was shaken for $8 \mathrm{~h}$ at RT. Then the films were removed and rinsed three times. All the washed dye solution was collected and finally diluted to a constant volume of $250 \mathrm{~mL}$. The fluorescence intensity of the solution was calculated and the carboxyl group density at the film surface was measured by comparing the detected values of the washing solution with that of the initial dye.

\section{Grafting amines to the PU-1 film}

The PU-1 film was immersed in an acetonitrile solution of 2propynylamine, 2-azido-ethylamine or PFPA-amine $2(50 \mathrm{mM}$, $20 \%$ ethanol was added in the case of preparing solution 2 ) and shaken continuously overnight at RT. Afterward, the film sample was rinsed thoroughly with ethanol, air dried in the dark and stored at $4{ }^{\circ} \mathrm{C}$ until use. The resulting films were named PU-1-alkyne, PU-1-azide, and PU-1-PFPA respectively.

\section{Click linkage between PU-1-alkyne and dansyl azide 5}

At RT, PU-1-alkyne was immersed in the dansyl azide $\mathbf{5}$ solution $\left(0.01 \mathrm{M}, t-\mathrm{BuOH}-\mathrm{H}_{2} \mathrm{O}=1: 1\right)$, and $\mathrm{Na}$ ascorbate $(40 \% \mathrm{~mol})$ and $\mathrm{Cu}^{2+}(10 \% \mathrm{~mol})$ were added to initiate the click reaction. After $2 \mathrm{~h}$, the sample was removed, rinsed with water and ethanol, and finally immersed in ethanol with continuous shaking overnight to remove the physically adsorbed fluorescent dye. The control sample was produced using the same procedure except that $\mathrm{Cu}^{2+}$ was not added.

\section{Photo attaching heparin to PU-1-PFPA}

Heparin solution (2.5 $\mathrm{mg} \mathrm{mL}^{-1}$, sodium salt) was cast on PU-1PFPA and photoreaction was performed in wet conditions for 5 min. Excess heparin was then removed and the resultant film was rinsed thoroughly with DI water. The heparin attached film (PU-1-PFPA-heparin) was air dried in the dark before the static contact angle measurement, XPS analysis, and platelet adhesion test. The process was repeated using recycled heparin solution and the obtained film (PU-1-PFPA-heparin-R, R = recycled) was used to test platelet adhesion properties.

\section{Quantification of immobilized heparin}

The photo-attachment of heparin was determined by titration as previously reported. ${ }^{37}$ The calibration curve was determined as follows. To $3 \mathrm{~mL}$ toluidine blue solution (stock solution, $25 \mathrm{mg}$ in $0.01 \mathrm{~N}$ hydrochloric acid containing $0.2 \mathrm{wt} \% \mathrm{NaCl}$ ), a known amount of aqueous heparin solution $(2 \mathrm{~mL})$ was added. The mixture was agitated using a vortex mixer, then $n$-hexane ( $3 \mathrm{~mL}$ ) was added and the mixture was shaken well to extract the toluidine blue-heparin complex into the organic layer. The toluidine blue remaining in the aqueous phase was thus determined by absorption at $631 \mathrm{~nm}$. The linear relationship between the absorbance of residual toluidine blue at $631 \mathrm{~nm}$ and the concentration of heparin in aqueous solution was obtained as a curve. Next, the amount of immobilized heparin was determined. Toluidine blue solution $(3 \mathrm{~mL})$ was mixed with an aqueous solution $(2 \mathrm{~mL})$, and PU-1-PFPA-heparin $(1 \times 1 \mathrm{~cm})$ was immersed in the mixed solution for $30 \mathrm{~min}$. $n$-Hexane ( $3 \mathrm{~mL}$ ) was then added to ensure uniformity of the treatment. The absorbance $(631 \mathrm{~nm})$ of the aqueous layers of the solution was checked and the amount of immobilized heparin was thus calculated from the calibration curve.

\section{Platelet adhesion test}

The platelet adhesion test was carried out in a static blood environment according to the literature. ${ }^{38}$ Human whole blood was collected from healthy volunteers and mixed with citrate solution (10\% volume, concentration $3.8 \%$ ) as an anticoagulant. The blood was centrifuged at $1200 \mathrm{rpm}$ for $10 \mathrm{~min}$ at RT to obtain platelet-rich plasma (PRP). The number of platelets was determined using a haemocytometer. The platelet concentration of PRP solution was adjusted to $1 \times 10^{5}$ cells per $\mu \mathrm{L}$ by adding phosphate buffered saline (PBS, $\mathrm{pH}$ 7.4) to PRP.

PU-1-PFPA-heparin, PU-1-PFPA-heparin-R and untreated PU samples $\left(1 \times 2 \mathrm{~cm}^{2}\right)$ were equilibrated with PBS (pre-warmed to $37{ }^{\circ} \mathrm{C}$ ) for $1 \mathrm{~h}$ and then immersed in $1 \mathrm{~mL}$ of the separated platelet suspension for $2 \mathrm{~h}$ at $37{ }^{\circ} \mathrm{C}$ with $5 \% \mathrm{CO}_{2}$. After incubation, the samples were taken out and gently washed three times with PBS to remove the non-adhered platelets. Quantification of platelet adhesion on the samples was carried out according to the following equation:

$$
\text { platelet adhesion }(\%)=\left(N-N_{\mathrm{t}}\right) / N \times 100
$$

where $N$ is the number of platelets in PRP solution before contact and $N_{\mathrm{t}}$ is the number of platelets in PRP solution after contact.

\section{Copper-free click study of PFPA}

Model REACTION. Compound $4(20 \mathrm{mg}, 0.08 \mathrm{mmol})$ was dissolved in propargyl alcohol $(0.3 \mathrm{~mL}, 5.2 \mathrm{mmol})$ and the solution was left to stand for $40 \mathrm{~min}$ before being checked by TLC. Then the reaction solution was evaporated at RT and purified by column chromatography to give a white solid which was submitted to ${ }^{1} \mathrm{H}$ NMR and HRMS analyses. Meanwhile, another model reaction was performed by dissolving $4(20 \mathrm{mg}$, $0.08 \mathrm{mmol}$ ) and propargyl alcohol $(0.03 \mathrm{~mL}, 0.52 \mathrm{mmol})$ in $0.4 \mathrm{~mL}$ mixed solvent $\left(t-\mathrm{BuOH}-\mathrm{H}_{2} \mathrm{O}=1: 1\right)$ containing $40 \% \mathrm{~mol}$ $\mathrm{Na}$ ascorbate and $10 \% \mathrm{~mol} \mathrm{CuSO}_{4}$ (calculated based on 4). The reaction mixture was continuously shaken for $2 \mathrm{~h}$ and the product was also purified by column chromatography. A proton NMR spectrum of the obtained white solid was then collected. 
COPPER-FREE CLICK REACTION ON PU-1-PFPA. PFPA-amine 2 was first coupled to PU-1-s to give the corresponding PU-1-PFPAs. Two pieces of PU-1-PFPA-s films was then immersed in 2,2methylbut-3-yn-1-amine overnight at RT and $37^{\circ} \mathrm{C}$, respectively. Next, all the films were rinsed thoroughly with ethanol before being stained with $0.01 \mathrm{~g} \mathrm{~mL}^{-1}$ Acid Orange II at $\mathrm{pH} 3$ with continuous shaking overnight. ${ }^{39}$ The stained sample films were rinsed with distilled water followed by $1 \mathrm{mM} \mathrm{HCl}$ to remove adsorbed dye molecules before taking the images.

At the same time, PU-1-PFPA $\left(0.5 \times 0.5 \mathrm{~cm}^{2}\right)$ was immersed in $0.5 \mathrm{~mL}$ liquid 7 overnight at RT. The obtained film was then thoroughly rinsed with ethanol before submitting to XPS analysis. As a control, PU was also treated with 7 by the same approach before XPS analysis.

\section{Results and discussion}

\section{Photografting of $p$-azidotetrafluorobenzoic acid (3) onto PU}

p-azidotetrafluorobenzoic acid (3) was synthesized as a model compound and photografted on PU to test the photo-coupling feasibility as well as to determine the photografting efficacy. In ATR spectra (Fig. S1 $\dagger$ ), the peak $1703 \mathrm{~cm}^{-1}$ (ascribed to the stretch of $\mathrm{C}=\mathrm{O}$ in carboxylic acid and ester) intensified after photoreaction, suggesting the presence of 3 on the modified PU (PU-3). Meanwhile, PU-3 was stained with THA, a cationic dye that can form ionic bonds with carboxylic acid groups via electrostatic interaction. After thorough rinsing, a uniform blue color on the modified circular area was visible (Fig. 1(c)). As depicted in Fig. 1(e), F 1-s signal in the XPS survey of PU-3 further confirmed the attachment of $\mathbf{3}$ on PU, since F element existed only in $\mathbf{3}$ and not in the untreated PU. The conversion ratio $(\chi)$ was calculated to be $53.0 \%$ based on XPS data (Fig. $S 2 \dagger$ ), meaning 3 was grafted onto the PU film at a density of 0.53 molecule of 3 per PU repeating unit (assuming two nitrogen atoms in every repeating unit). The introduction of a carboxylic acid group manifested the change of a macroscopic property - hydrophilicity. The static contact angle of water decreased from 97.6 (untreated PU) to $81.5^{\circ}$ (PU-3) as shown in Fig. 1(a) and (b). The newly introduced carboxylic group makes the film more hydrophilic.

According to the colorimetric titration method, ${ }^{36}$ the surface carboxylic group density on PU-3 was determined to be $10.3 \pm$ $1.6 \mathrm{nmol} \mathrm{cm}{ }^{-2}$. These data are slightly higher compared with the carboxylic group density $\left(4.0 \mathrm{nmol} \mathrm{cm}{ }^{-2}\right.$ ) generated on the poly(ether imide) surface using a thermochemical reaction. ${ }^{40}$ Atomic force microscopy (AFM) was also performed to evaluate the possible morphological alterations after the UV irradiation process. As shown in Fig. S3, $\uparrow$ the untreated PU film possesses a smooth surface with a root mean square roughness $\left(R_{\mathrm{q}}\right)$ of $8.8 \mathrm{~nm}$, while PU-3 shows a bumpy surface with a higher $R_{\mathrm{q}}$ $(31.5 \mathrm{~nm})$. The increased roughness seems to be mainly caused by the surface photoreaction since the control PU film (which had gone through the similar treatment as PU-3 except without the presence of 3 ) has a $R_{\mathrm{q}}$ of only $12.5 \mathrm{~nm}$. The fact that model compound 3 is present on a rough surface with a larger surface area might contribute to the higher surface carboxylic group density.

\section{Fabrication of PU-1-PFPA as a SPP}

It is usually necessary to confer reactive species both on solid surfaces and on target cargoes for coupling. However, it is challenging to chemically derivatize functional molecules such as carbohydrates. The photosensitive PFPA groups, once immobilized on solid surfaces, have proven efficient in photocapturing carbohydrates with preserved bioactivity. ${ }^{\mathbf{4 1 , 4 2}}$ Similarly, PS simple polymer and PS nano-particles have been photografted on solid surfaces based on PFPA photochemistry. ${ }^{\mathbf{4 3 - 4 5}}$ Therefore, we first fabricated PU-1 using an approach similar to the fabrication of PU-3 and subsequently coupled 2 onto PU-1 to produce the photoactive PU-1-PFPA as depicted in Scheme 1. To validate the introduced phenyl azide group, ATR spectra were

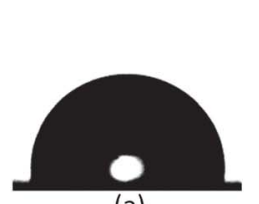

(a)

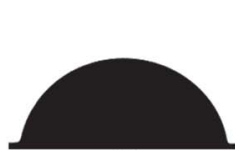

(b)

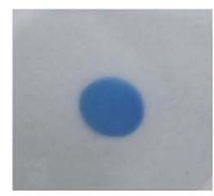

(c)
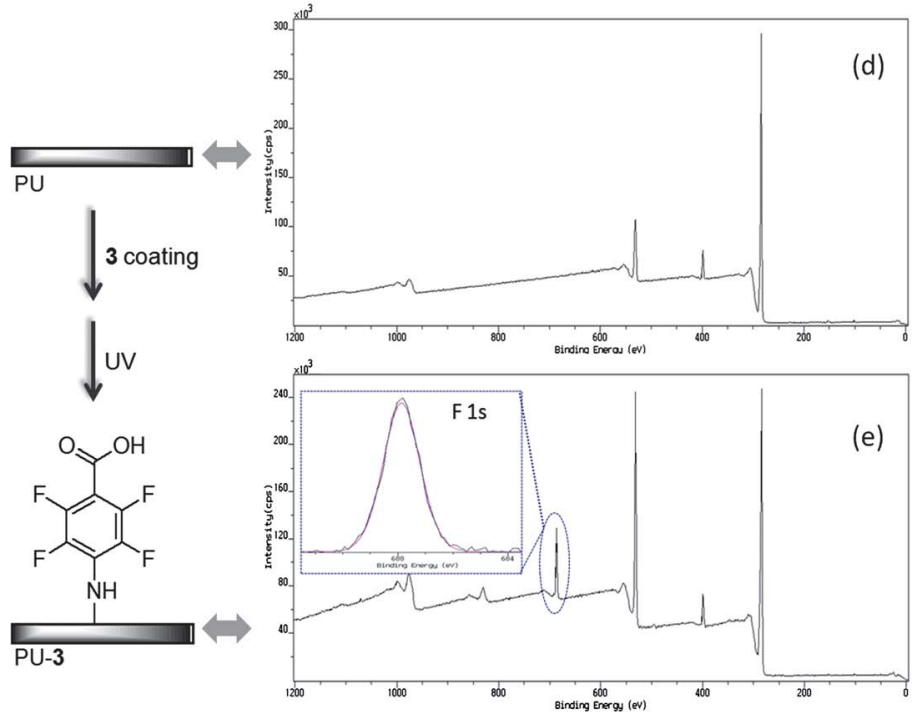

Fig. 1 Characterization of PU-3. Contact angle measurements of PU (a) and PU-3 (b); visualization of THA dye stained PU-3 (c); XPS survey of PU (d) and PU-3 (e). 


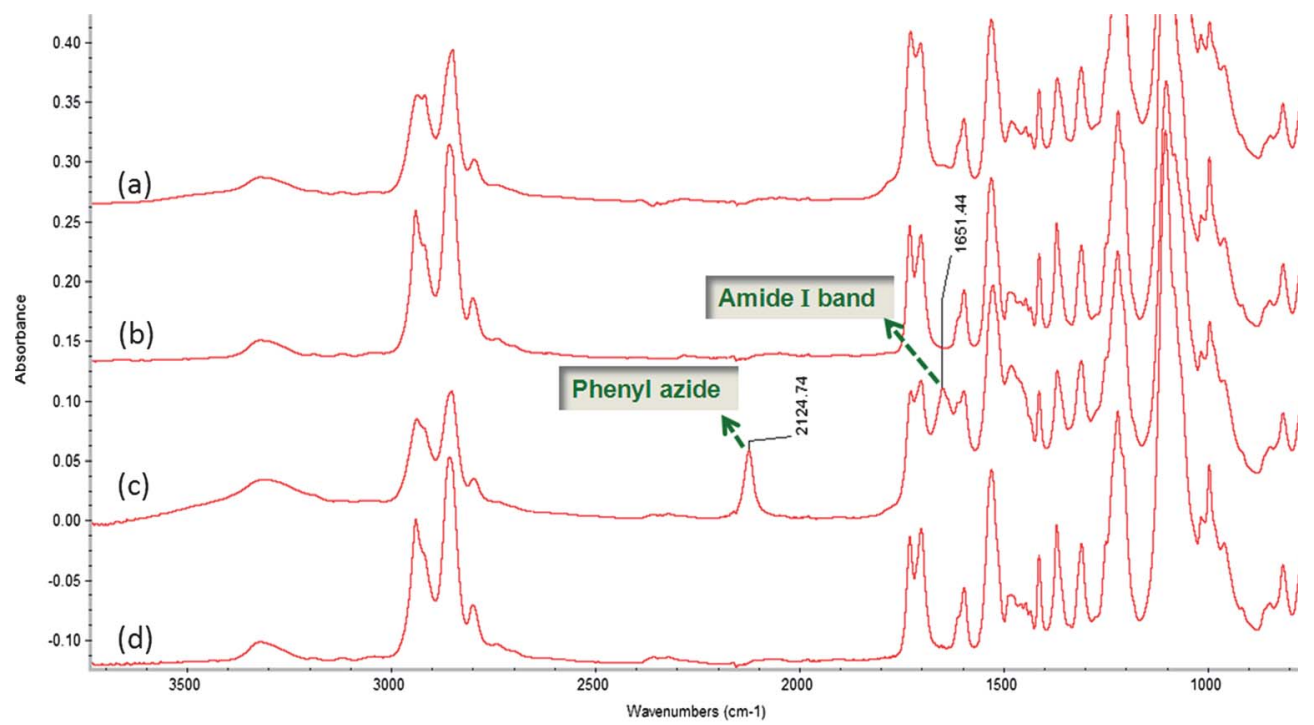

Fig. 2 ATR spectra of (a) untreated PU; (b) PU-1; (c) PU-1-PFPA; (d) PU-1-PFPA (back of the film).

collected and are presented in Fig. 2. The spectrum of PU-1PFPA (Fig. 2(c)) presented a new peak at $2124.7 \mathrm{~cm}^{-1}$ (ascribed to $-\mathrm{N}_{3}$ of PFPA) as compared with that of PU-1 (Fig. 2(b)). There was no detectable $-\mathrm{N}_{3}$ peak on the back of PU-1-PFPA, which means that the physically adsorbed 2 could be removed by rinsing. At the same time, another peak $\left(1654.44 \mathrm{~cm}^{-1}\right)$ was observed, which corresponds to the amide bond that was newly formed in PU-1-PFPA. The differences between the ATR spectra of PU-1, PU-1-PFPA and PU-1-PFPA (back) demonstrated that 2 was chemically conferred onto PU-1.

\section{Functionalization of PU-1-PFPA with heparin}

On the PU-derived SPP (PU-1-PFPA), heparin was covalently attached via the photo-coupling method. After a thorough rinse, the resultant sample PU-1-PFPA-heparin was air dried overnight before the measurement of the static contact angle. The contact angle distinctly decreased from $90.0 \pm 0.7^{\circ}$ (PU-1-PFPA) to $78.3 \pm 0.3^{\circ}$ (PU-1-PFPA-heparin) after the attachment of hydrophilic heparin. To further confirm the attachment, PU-1PFPA-heparin was subjected to XPS analysis and the S 2p signal was detected (Fig. 3). Since S uniquely exists in heparin, the observed S 2p signal suggests the successful attachment of heparin on PU-1-PFPA. Meanwhile, the F atomic concentration decreased significantly from $3.40 \%$ to $0.68 \%$ (Fig. 3), which is reasonable considering that once PU-1-PFPA is covered with heparin (F-free polymer) as a result of photolysis, many of the $\mathrm{F}$ atoms of PFPA might be buried to a depth undetectable by XPS.

As the first event of blood clotting cascades, platelet adhesion has been the accepted evaluator for the hemocompatibility of blood contacting medical devices. As shown in Fig. 4, the percentage of platelet adhesion on the untreated PU film reached $43.1 \pm 2.2 \%$, a high level of coagulation. After the attachment of heparin, the platelet adhesion reduced significantly to $17.6 \pm 0.8 \%$, indicating that PU-1-PFPA-heparin effectively prevented platelet aggregation and adsorption.
The heparin density on PU-1-PFPA-heparin was determined by a colorimetric toluidine blue assay to be $0.64 \pm 0.08 \mu \mathrm{g}$ $\mathrm{cm}^{-2}$, which is comparable to that achieved on other substrates such as a polysulfone membrane $\left.(0.86 \mu \mathrm{g} \mathrm{cm})^{-2}\right)^{46}$ and a silicone surface $\left(0.68 \mu \mathrm{g} \mathrm{cm}{ }^{-2}\right) \cdot{ }^{47}$ Usually higher heparin density can be achieved by adopting polymeric spacers, such as chitosan $\left(1.37-2.97 \mu \mathrm{g} \mathrm{cm}^{-2}\right)^{27}$ and polyallylamine (3.6-4.1 $\mu \mathrm{g}$ $\left.\mathrm{cm}^{-2}\right){ }^{48}$ Polymeric spacers provide a beneficial reaction microenvironment with less steric hindrance and more accessible bonding sites for heparin coupling. In comparison with the PU substrate grafted with chitosan spacer and heparin (PU-chitosan-heparin), ${ }^{27}$ PU-1-PFPA-heparin achieved a similar platelet adhesion reduction level (from $43.1 \pm 2.2 \%$ to $17.6 \pm 0.8 \% v s$. from $27 \%$ to $13 \%$ ) with only half the surface heparin concentration $\left(0.64 \pm 0.08 \mu \mathrm{g} \mathrm{cm}^{-2}\right.$ vs. $\left.1.37 \mu \mathrm{g} \mathrm{cm} \mathrm{cm}^{-2}\right)$. This is probably due to the fact that the photo-coupling method to immobilize heparin is quite different from the coupling methodology based on carbodiimide chemistry. In the latter case, excess EDC (70 equiv.) is usually used ${ }^{27,46,49}$ to ensure the coupling efficacy. Given that stoichiometry, all the carboxylic groups on the heparin chain are activated. As a result, when the polysaccharide chain with multiple activated binding sites is exposed to surface amines, more than one activated carboxylic acid groups in the same heparin chain may couple to the polymer surface, leading to multipoint attachment and relatively rigid heparin chains (Fig. S4†). For the photo-coupling method, when the hydrophobic surface of PU-1-PFPA (contact angle $90.0 \pm 0.7^{\circ}$ ) contacts the heparin solution, it is likely that very small amounts of heparin molecules lay themselves completely on the PU-1-PFPA surface so that few heparin chains are captured via multi $\mathrm{N}-\mathrm{C}$ bonds (Fig. S4†). The photo-coupling method under wet conditions probably produced free polysaccharide chains that seemed to stand on the PU-1-PFPA surface, supporting the fact that matchable platelet adhesion reduction was achieved despite a reduced heparin density. 


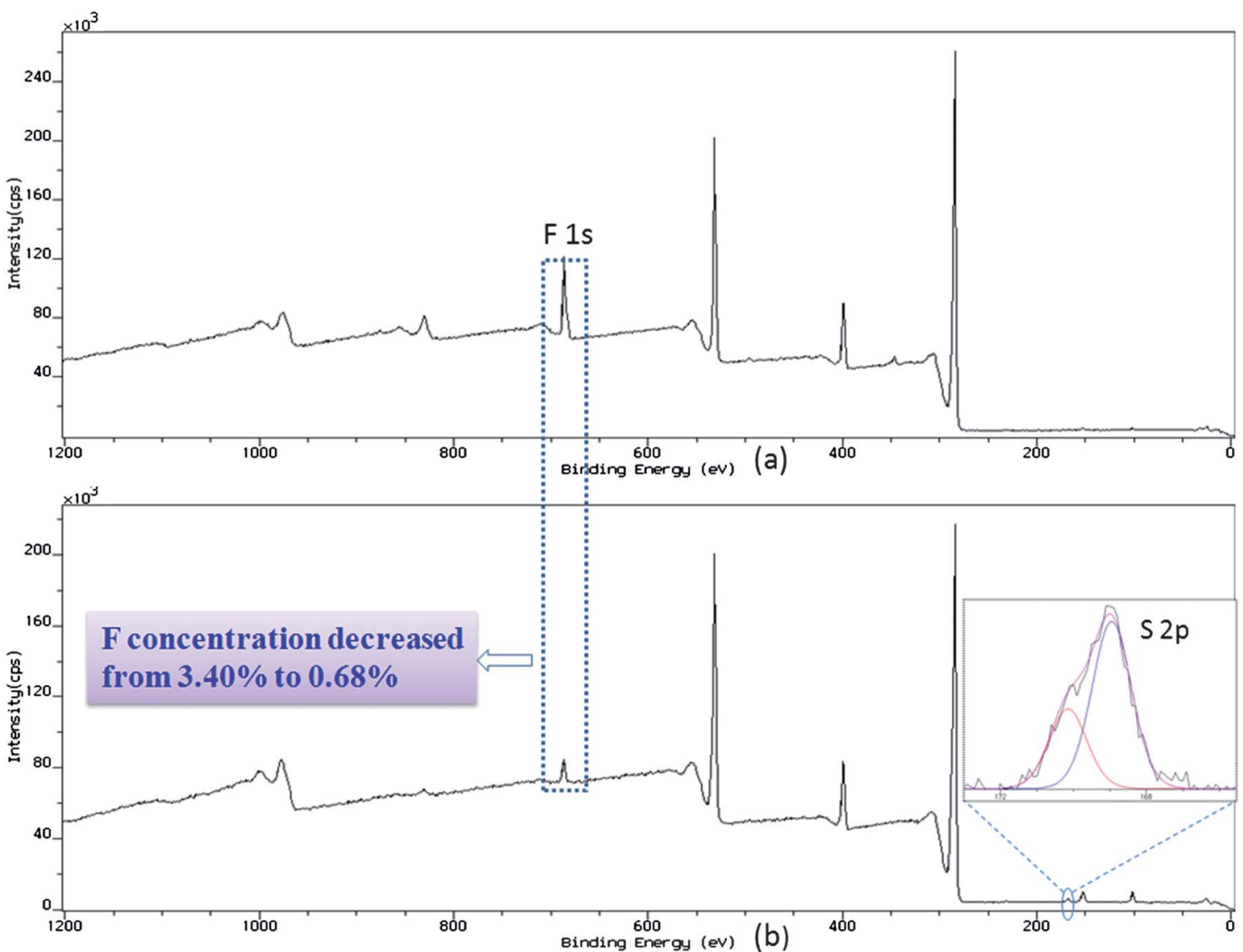

Fig. 3 XPS survey of (a) PU-1-PFPA and (b) PU-1-PFPA-heparin.

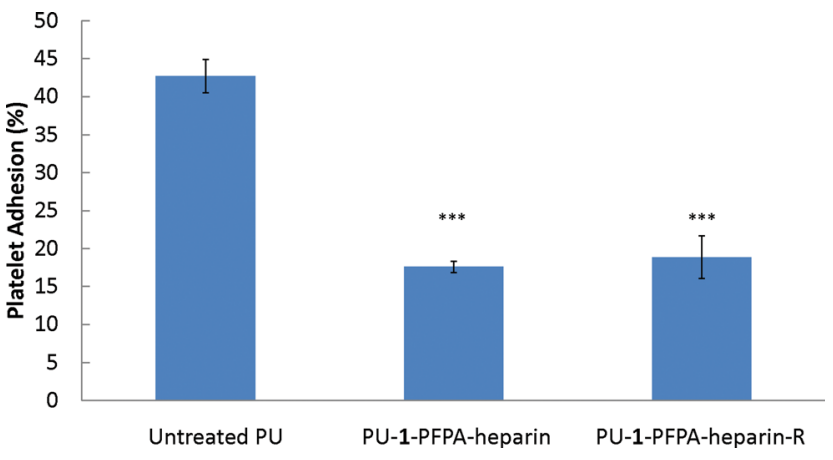

Fig. 4 Quantification of platelet adhesion on untreated PU, PU-1-PFPA-heparin and PU-1-PFPA-heparin- $R$ ( $R=$ recycled). The diagram includes $t$-test results $(n=$ 3) with respect to untreated $\mathrm{PU}\left({ }^{*} p<0.05,{ }^{* *} p<0.01\right.$ and $\left.{ }^{* *} p<0.001\right)$.

The photo-attaching process was performed in wet conditions, and only those heparin molecules in covalent bonding distance with the surface generated nitrenes could be captured, leaving the remaining heparin molecules intact in the aqueous solution. Hence, it might be possible to recycle the vast majority of heparin in the solution, and retain the antithrombotic activity. To test this hypothesis, the heparin solution that had been exposed to UV irradiation during the photografting process was salvaged (by pipette tips) and again cast on PU-1-PFPA for another grafting process to produce the modified film PU-1-PFPA-heparin-R ("R" refers to recycled).

Interestingly, the percentage of platelet adhesion on the film immobilized with recycled heparin - PU-1-PFPA-heparin-R was only $18.9 \pm 2.8 \%$, which is not significantly different from that on PU-1-PFPA-heparin (17.6 $\pm 0.8 \%$, Fig. 4$)$. By comparing the weight of recycled heparin solution to that of the initially casted heparin solution, we estimate the recovery to be no less than $94 \%$. This indicates that the PFPA photo-coupling strategy for heparin grafting is more economical than the EDC/NHS coupling method where excess heparin with all carboxylic acid groups pre-activated is usually required for heterogeneous immobilization on a solid surface.

The first advantage of PU-derived SPP is that a great variety of molecules, regardless of the molecular structure or architecture, could be immobilized by this simple and fast photo-activation procedure as long as they can endure UV irradiation for a few minutes. Second, the photo-grafting efficacy can be conveniently controlled simply by adjusting the light intensity. ${ }^{13}$ Spatial specific immobilization is also possible using photomasks during UV irradiation. ${ }^{15}$ Third, since phenyl azide derivatives have been photo-immobilized onto various substrate materials such as polyimide ${ }^{50}$ polystyrene polypropylene, ${ }^{51}$ and even inorganic carbon materials, ${ }^{15}$ this two step general modification method (PU $\rightarrow$ PU-1 $\rightarrow$ PU-1-PFPA) to produce SPP could readily be extended to these substrate materials. 


\section{Fabrication of a surface clickable platform (SCP)}

As discussed above, the SPP of PU-1-PFPA is capable of directly capturing various molecules upon UV irradiation, but the methodology is not applicable to those light sensitive functionalities that need to be conferred on the surface. As such, universal platforms that could achieve the attachment of functional moieties via the robust and versatile linkage methodology such as the copper-catalyzed azide-alkyne cycloaddition (CuAAC, also named "click" reaction) are also desirable. In principle, direct photo-coupling of PFPA-alkyne or PFPA-azide derivatives onto the PU surface is the most effective modification method. However, the PFPA-alkyne derivative can undergo a self-click reaction ${ }^{52}$ even during purification which is problematic. On the other hand, alkyl azides could also undergo photodecomposition when exposed to UV irradiation. ${ }^{53}$ Hence we herein attached 2-azidoethylamine and 2-propynylamine on PU-1 via a two-step method to produce SCP as PU-1-azide and PU-1-alkyne, respectively (Fig. 5).

The ATR spectrum of PU-1-azide presented an expected azide peak (2102.15 $\mathrm{cm}^{-1}$ ) (Fig. S5†), suggesting the successful covalent attachment of 2-azidoethylamine on PU-1. The azide peak shifted from $2124.7 \mathrm{~cm}^{-1}$ (PU-1-PFPA) to $2102.2 \mathrm{~cm}^{-1}$ (PU-1-azide). The higher wavenumber of the azide peak in PU-1-PFPA is probably due to the electron withdrawing effect of the perfluorophenyl group. As presented in Fig. 5(a) and (b), high-resolution XPS $\mathrm{N}$ 1s spectrum was curve-fitted and a distinct $\mathrm{N}$ 1s peak was resolved at a binding energy of $404.5 \mathrm{eV}$, which is attributed to the central nitrogen of the azide moiety. The other two nitrogen atoms of the azide moiety possess lower binding energies ${ }^{54}$ and the corresponding peaks are consequently hidden in the main peak of amide nitrogen $(400.4 \mathrm{eV})$. The satellite $\mathrm{N}$ 1s peak confirms the successful attachment of 2-azidoethylamine on PU-1. To validate the surface alkynyl groups, we next integrated a dansyl-azide $5^{35}$ onto PU-1-alkyne via the CuAAC chemistry method. After thorough rinsing, a uniform green fluorescence was observed (Fig. 5(d)), while only blue autofluorescence was seen on PU-1-alkyne (Fig. 5(c), the control without the addition of $\mathrm{Cu}^{2+}$ ). This result indicates the successful formation of uniformly distributed alkynyl groups on the surface of PU-1-alkyne.

With the established SCP of PU-1-alkyne/PU-1-azide, a variety of functional reagents containing bioorthogonal tags could be conveniently conferred via the CuAAC chemistry method. To this end, PU-1-azide/PU-1-alkyne could be regarded as a mutual supplement to PU-1-PFPA in that the former provides a robust and versatile bonding method to immobilize alkynyl/azido cargoes with light-sensitive functionalities, while the latter is capable of readily integrating various light-insensitive functionalities without any chemical structural modification.

\section{Copper-free click performance of PFPA}

CuAAC has found numerous applications in drug design, ${ }^{55}$ chemical biology ${ }^{56}$ and materials science, ${ }^{57}$ but entails the use of a copper salt catalyst, which exhibits in vivo toxicities in biological system applications. ${ }^{58}$ To this end, the strain-promoted azide-alkyne cycloadditions (SPAAC) have been developed as a copper-free alternative with suitable reactivity for bioorthogonal chemistry applications..$^{\mathbf{5 9 6 0}}$ However, the preparation of strained cycloalkynes usually requires extensive and laborious synthetic work, so this method is rarely appropriate for straightforward synthetic applications. Therefore, the development of a simple version of copper-free cycloaddition reaction is highly appreciated.

To our knowledge, the first copper-free cycloaddition between PFPA and alkynes was mentioned by Yan and

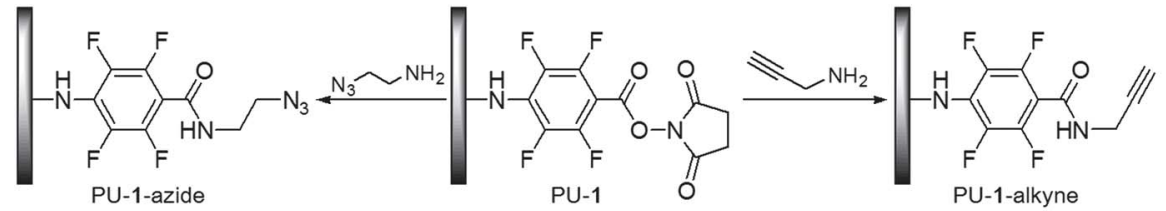

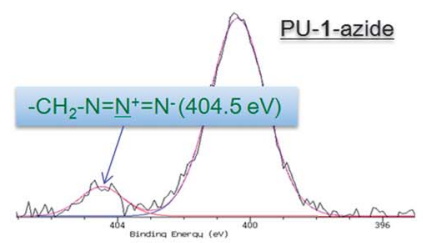

(a)

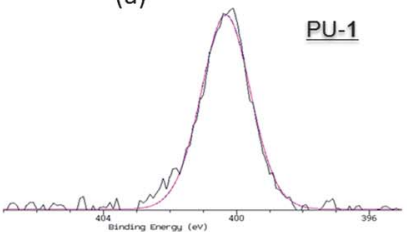

(b)

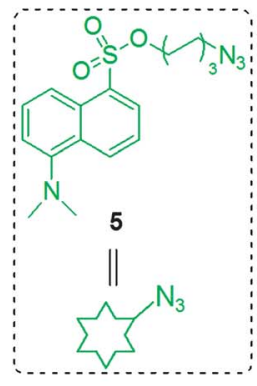

Fig. 5 Formation and characterization of a surface clickable platform (SCC). High-resolution XPS N 1s spectra of PU-1-azide (a) and PU-1 (b); visualization of PU-1alkyne (c) and PU-1-alkyne-5 (d). 
coworkers, ${ }^{52}$ but no further details have been presented. We initially attempted to prepare prop-2-ynyl 4-azido-2,3,5,6-tetrafluorobenzoate (8) by esterification of 3 with propargyl alcohol (Fig. S6 + ). The derivative 8 contains the shortest linker $-\mathrm{CH}_{2}-$, probably decreasing the crosslinking possibility during the photo-coupling process. However, self-click reactions proceeded in the purification process (see Fig. S6†). Based on the observation on thin layer chromatography (TLC), we could draw a tentative conclusion that the self-click reaction is concentration dependent and thermally promoted.

We then investigated the similar potential copper-free click behaviour of PFPA groups on PU-1-PFPA. First, a model reaction was performed in an open centrifuge tube by mixing diluted solutions of $4\left(0.15 \mathrm{~mol} \mathrm{~L}^{-1}\right)$ and $6\left(0.15 \mathrm{~mol} \mathrm{~L}^{-1}\right)$ at $\mathrm{RT}$ and $37{ }^{\circ} \mathrm{C}$, respectively. The reaction process was monitored by TLC. No new visible spot was observed (data not shown) on the TLC plates for both samples within $40 \mathrm{~min}$; however, a new spot appeared on the baseline (Fig. S6†) after the solvent evaporated overnight. The new spot that formed at $37{ }^{\circ} \mathrm{C}$ seemed intensified in comparison with the spot produced at RT. These observations indicate that the copperfree click between $\mathbf{4}$ and $\mathbf{6}$ is concentration dependent and thermo-promoted, which is in accord with the self-click of $\mathbf{8}$. To further test this copper-free click hypothesis, we next directly dissolved 4 in propargyl alcohol to find that a new spot was observed on the TLC plate within 40 min (Fig. 6). The reaction mixture was submitted to HRMS analysis and a new peak with $[\mathrm{M}+\mathrm{H}]^{+}=306.0511$ (calculated 306.0502) was detected, which corresponded to the newly produced triazole adduct. Meanwhile, the CuAAC reaction between 4 and propargyl alcohol was also performed at RT. The ${ }^{1} \mathrm{H}$ NMR spectra of the purified product $\mathbf{9}$ and copper-free click adduct (isomer mixture) were collected. Based on the analysis of triazole-H integration, it could be concluded that the copper-free click reaction between $\mathbf{4}$ and propargyl alcohol afforded a mixture of 1,4- and 1,5-regioisomers with a ratio of ca. $4: 1$ (0.999 : 0.249) (Fig. 6).
As subsidiary evidence, 2-azidoethyl benzoate, the synthetic alkyl-azide, ${ }^{61}$ was dissolved in propargyl alcohol at RT, but no visible reaction was observed on the TLC plate within $40 \mathrm{~min}$, further suggesting that the copper-free click reaction between 4 and propargyl alcohol is specifically due to the presence of the PFPA moiety.

Subsequently, the feasibility of copper-free click reaction on PU-1-PFPA was tested. Initially, PU-1-PFPA-s ( $\mathrm{s}=$ spotting) was fabricated to be treated with propiolic acid to confer carboxylic acid groups that could be stained by THA. However, PU-1-PFPA was found to be readily dissolvable in propiolic acid. We then turned to 2,2-methylbut-3-yn-1-amine, a liquid alkyne-amine, in which PU-1-PFPA-s was immersed overnight in a dark place (at RT and $37^{\circ} \mathrm{C}$ respectively). After being stained with Acid Orange II, the casting region (PU-1-PFPA) for both samples exhibited a distinctly deeper brownish color (Fig. 7(a) and (b)) in contrast to the unspotted area on the films. No drastic difference was observed between the samples clicked at RT (Fig. 7(a)) and $37^{\circ} \mathrm{C}$ (Fig. 7(b)). This observation indicates that the copper-free cycloaddition between PU-1-PFPA and 2,2-methylbut-3-yn-1amine proceeded smoothly in the absence of the copper catalyst at RT. To further confirm this spontaneous click reaction, PU-1PFPA was treated with 7, a liquid alkyne containing chlorine atoms. As expected, a $\mathrm{Cl} 2 \mathrm{p}$ signal was detected in the XPS spectra (Fig. 7(c) and (d)) while no Cl 2p peak was observed on the back of the resultant film (PU-1-PFPA-7, Fig. 7(e)) and the control sample (PU, data not shown). Meanwhile, the highresolution $\mathrm{N}$ 1s spectra were curve-fitted and resolved at the binding energy of $401.7 \mathrm{eV}$ and $400.5 \mathrm{eV}$ with a ratio of $1: 2$ (Fig. 7(f) and (g)), which are attributed to the nitrogen atoms of the newly formed triazole adduct. ${ }^{35}$ All the evidence collectively supports the feasibility of copper-free click of alkynes onto the surface of PU-1-PFPA.

Metal free cycloaddition of azide with alkynes usually remains sluggish due to the relatively high kinetic-energy barrier (ca. $26 \mathrm{kcal} \mathrm{mol}^{-1}$ ), ${ }^{62}$ but the reaction could be promoted by prolonged heating, resulting in a mixture of $1,4-$ and

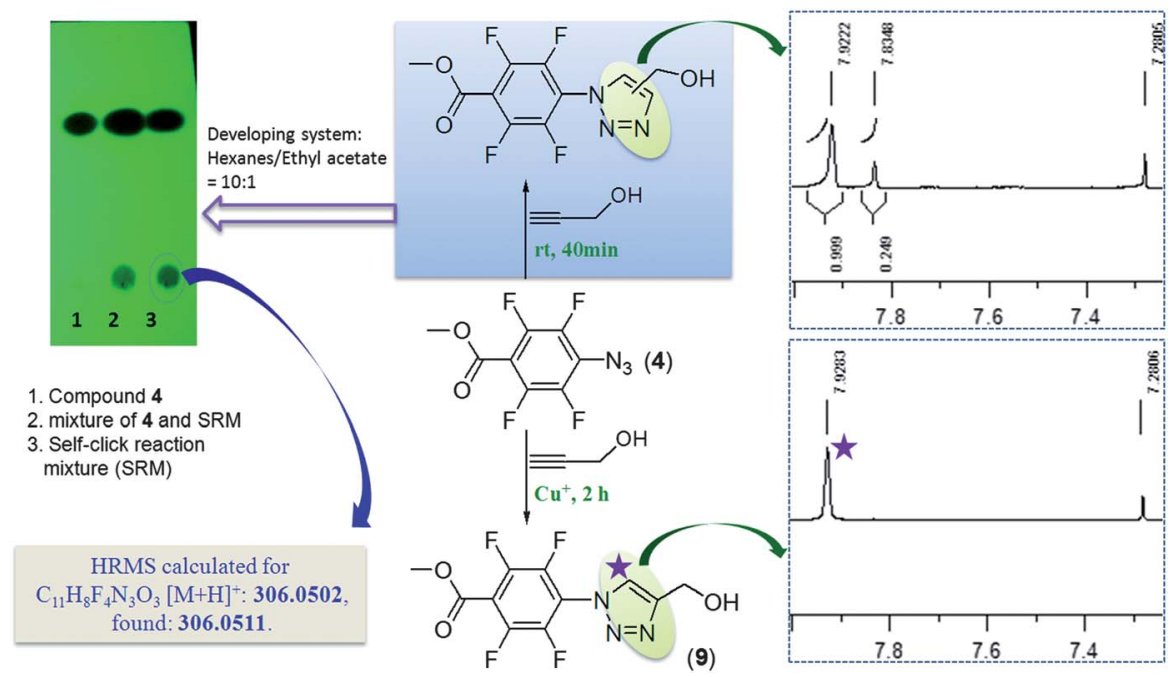

Fig. 6 Model study of copper-free click reactions between the PFPA methyl 4-azido-2,3,5,6-tetrafluorobenzoate (compound 4) and the alkyne prop-2-yn-1-ol. 


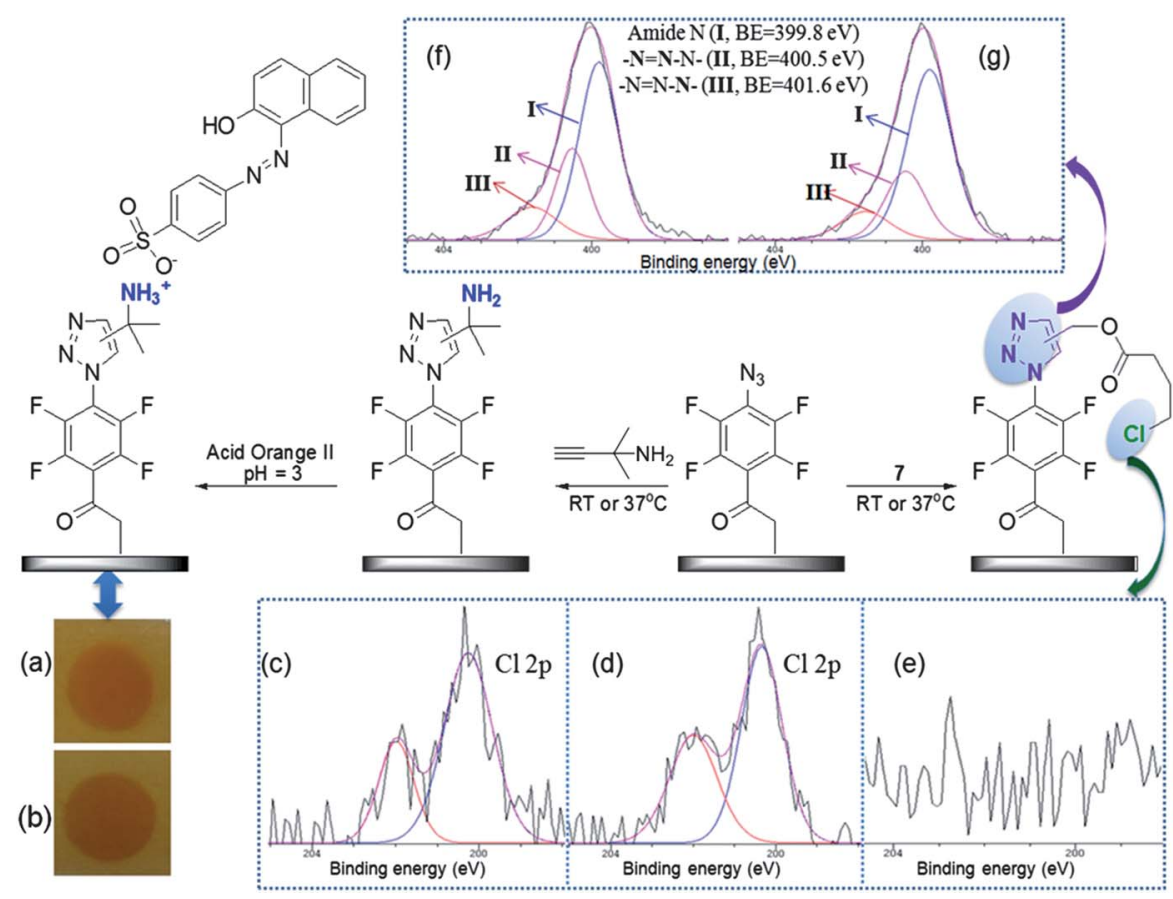

Fig. 7 Study of copper-free click reactions on PU-1-PFPA. Acid Orange II staining of PU-1-PFPA samples copper-free clicked with 2,2-methylbut-3-yn-1-amine at (a) RT and (b) $37^{\circ} \mathrm{C}$ respectively; (c-e) high-resolution XPS Cl 2P spectra of PU-1-PFPA-7 (c and d: spectra for the films on which copper-free click reactions proceeded at $37^{\circ} \mathrm{C}$ and RT, respectively) and its back $\left(\mathrm{e}, 37^{\circ} \mathrm{C}\right)$; $(\mathrm{f}-\mathrm{g})$ high-resolution XPS N 1s spectra of PU-1-PFPA-7 films obtained from copper-free click reactions proceeded at $37{ }^{\circ} \mathrm{C}$ and $\mathrm{RT}$, respectively.

1,5-regioisomers. In contrast, copper(I) catalysis dramatically accelerated the click reaction between azides and terminal alkynes to give excellent yields of 1,2,3-triazole. ${ }^{63}$ An alternative to CuAAC is SPAAC, which possesses low activation energy $(\Delta E=$ $8.0 \mathrm{kcal} \mathrm{mol}^{-1}$ ) compared to the strain free alkynes cycloaddition $\left(\Delta E=16.2 \mathrm{kcal} \mathrm{mol}^{-1}\right){ }^{64} \mathrm{Ni}$ and coworkers ${ }^{65}$ reported a copper-free click reaction between PFPA and phenyl alkynes in the crystalline phase due to the optimal spatial arrangement of the phenyl alkyne and the tetrafluorophenyl azide moiety, but the cycloaddition reaction under this condition proceeds slowly and requires 14 days for satisfactory conversion. Zhang and coworkers found that fluorinated benzenes are more aromatic than benzene in that the $\mathrm{p}_{z}$ lone pair electrons of $\mathrm{F}$ atom participate in the formation of hexagon $\pi$ current. ${ }^{66}$ On the other hand, due to the strong electronegativity of the $\mathrm{F}$ atom, the fluorinated benzene moiety of PFPA is a more electron-deficient structure. We speculated that the increased aromaticity and the electron deficiency probably decreased the activation energy for PFPA to achieve the transition state for feasible cycloaddition, leading to an obvious self-click phenomenon between PFPA and alkynes. The detailed kinetic data and the optimal reaction conditions for the copper-free cycloaddition between PFPA and alkynes will be investigated in other reports.

\section{Conclusions}

Using a two-step modification process based on PFPA photocoupling chemistry, we have successfully constructed a versatile and flexible surface platform PU-1-PFPA for both the immobilization of bioactive agents lacking reactive groups and the oriented immobilization of alkynyl functional moieties. Without any chemical derivatization, heparin was photocoupled to PU-1-PFPA with well preserved bioactivity. Heparin that remained ungrafted during the photocoupling process could be recovered and reused for another photo-attachment experiment on PU-1-PFPA to achieve the same level of anticoagulant activity as that of freshly prepared heparin solution. This work manifests the idea of combining photo-anchoring and capturing strategies based on PFPA chemistry to covalently immobilize functional moieties, especially those that cannot be readily derivatized, onto chemically inert thermoplastic polymeric substrates.

Meanwhile, it has been found that surface immobilized PFPA can undergo copper-free cycloaddition with alkynes in the interface even at RT, serving as a convenient and biologically benign conjugate methodology for achieving oriented grafting of bioactive reagents. In summary, the photoactive and copperfree clickable surface developed on the basis of PFPA photocoupling and click chemistry exemplifies facile tuning of the surface chemistry of chemically inert thermoplastic polymers for biomedical applications.

\section{Acknowledgements}

This work was supported by the Natural Sciences and Engineering Research Council of Canada (NSERC) Discovery grant (Grant no: RGPIN/372048-2009), the Manitoba Health Research Council (MHRC) Establishment grant, and Collaborative Health Research Projects (CHRP) operating grant (Grant no: CHRP 413713-2012). 


\section{References}

1 M. W. King, R. G. Guidoin, K. R. Gunasekera and C. Gosselin, Med. Prog. Technol., 1983, 9, 217.

2 M. Ikeuchi, R. Tane and K. Ikuta, Biomed. Microdevices, 2012, 14, 35.

3 S. C. Hsieh, C. M. Tang, W. T. Huang, L. L. Hsieh, C. M. Lu, C. J. Chang and S. H. Hsu, J. Biomed. Mater. Res., Part A, 2011, 99A, 576.

4 S. Hong, K. Y. Kim, H. J. Wook, S. Y. Park, K. D. Lee, D. Y. Lee and H. Lee, Nanomedicine, 2011, 6, 793.

5 A. Kishida, K. Mishima, E. Corretge, H. Konishi and Y. Ikada, Biomaterials, 1992, 13, 113.

6 G. Li, H. Liu, H. Zhao, Y. Gao, J. Wang, H. Jiang and R. I. Boughton, J. Colloid Interface Sci., 2011, 358, 307.

7 J. Wang, W. Hu, Q. Liu and S. Zhang, Colloids Surf., B, 2011, 85, 241.

8 M. H. Schneider, Y. Tran and P. Tabeling, Langmuir, 2011, 27, 1232.

9 K. Yamamoto, Y. Miwa, H. Tanaka, M. Sakaguchi and S. Shimada,J. Polym. Sci., Part A: Polym. Chem., 2002, 40, 3350.

10 H.-Y. Yu, J. Zhou, J.-S. Gu and S. Yan, J. Membr. Sci., 2010, 364, 203.

11 J. Li, D. Tan, X. Zhang, H. Tan, M. Ding, C. Wan and Q. Fu, Colloids Surf., B, 2010, 78, 343.

12 J. Brunner, H. Senn and F. M. Richards, J. Biol. Chem., 1980, $255,3313$.

13 L.-H. Liu and M. D. Yan, Acc. Chem. Res., 2010, 43, 1434.

14 B. M. Gorovits, A. P. Osipov, V. V. Doseeva and A. M. Egorov, Anal. Lett., 1991, 24, 1937.

15 L.-H. Liu, G. Zorn, D. G. Castner, R. Solanki, M. M. Lerner and M. Yan, J. Mater. Chem., 2010, 20, 5041.

16 L. Deng, O. Norberg, S. Uppalapati, M. Yan and O. Ramström, Org. Biomol. Chem., 2011, 9, 3188.

17 X. Wang, O. Ramström and M. D. Yan, J. Mater. Chem., 2009, 19, 8944.

18 H. Sigrist, H. Gao and B. Wegmuller, Biotechnology, 1992, 10, 1026.

19 Z. C. Pei, H. Yu, M. Theurer, A. Walden, P. Nilsson, M. D. Yan and O. Ramstrom, ChemBioChem, 2007, 8, 166.

20 H. C. Kolb, M. G. Finn and K. B. Sharpless, Angew. Chem., Int. Ed., 2001, 40, 2004.

21 J. P. Santerre, K. Woodhouse, G. Laroche and R. S. Labow, Biomaterials, 2005, 26, 7457-7470.

22 R. W. Matthew, B. Richard and K. Cay, Biomaterials, 2006, 27, 3608.

23 W.-H. Kuo, M.-J. Wang, C.-W. Chang, T.-C. Wei, J.-Y. Lai, W.-B. Tsai and C. Lee, J. Mater. Chem., 2012, 22, 9991.

24 A. Korematsu, Y. Takemoto, T. Nakaya and H. Inoue, Biomaterials, 2002, 23, 263-271.

25 J. Huang and W. Xu, Appl. Surf. Sci., 2010, 256, 3921.

26 Y. J. Du, J. L. Brash, G. M. Clung, L. R. Berry, P. Klement and A. K. C. Chan, J. Biomed. Mater. Res., Part A, 2007, 80, 216.

27 W. C. Lin, C. H. Tseng and M. C. Yang, Macromol. Biosci., 2005, 5, 1013.

28 Q. Lv, C. Cao and H. Zhu, J. Mater. Sci.: Mater. Med., 2004, 15, 607.
29 M. Yan, S. Cai, M. N. Wybourne and J. F. W. Keana, J. Am. Chem. Soc., 1993, 115, 814.

30 M. D. Yan, S. X. Cai, M. N. Wybourne and J. F. W. Keana, Bioconjugate Chem., 1994, 5, 151.

31 S. Attavar, M. Diwekar and S. Blair, Lab Chip, 2011, 11, 841. 32 J. F. W. Keana and S. X. Cai, J. Org. Chem., 1990, 55, 3640.

33 R. Rajagopalan, R. R. Kuntz, U. Sharma, W. A. Volkert and R. S. Pandurangi, J. Org. Chem., 2002, 67, 6748.

34 S. Angelos, Y. W. Yang, K. Patel, J. F. Stoddart and J. I. Zink, Angew. Chem., Int. Ed., 2008, 47, 2222.

35 L. Li, N. Zhao and S. Liu, Polymer, 2012, 53, 67.

36 V. B. Ivanov, J. Behnisch, A. Hollander, F. Mehdorn and H. Zimmermann, Surf. Interface Anal., 1996, 24, 257.

37 I.-K. Kang, O. H. Kwon, Y. M. Lee and Y. K. Sung, Biomaterials, 1996, 17, 841.

38 J. H. Lee, Y. M. Ju and D. M. Kim, Biomaterials, 2000, 21, 683. 39 P. Hamerli, Th. Weigel, Th. Groth and D. Paul, Biomaterials, 2003, 24, 3989.

40 R. Tzoneva, B. Seifert, W. Albrecht, K. Richau, T. Groth and A. Lendlein, J. Mater. Sci.: Mater. Med., 2008, 19, 3203.

$41 \mathrm{H}$. Wang, Y. Zhang, X. Yuan, Y. Chen and M. D. Yan, Bioconjugate Chem., 2011, 22, 26.

42 L.-H. Liu, H. Dietsch, P. Schurtenberger and M. D. Yan, Bioconjugate Chem., 2009, 20, 1349.

43 M. D. Yan, Chem.-Eur. J., 2007, 13, 4138.

44 T. Kubo, X. Wang, Q. Tong and M. D. Yan, Langmuir, 2011, 27, 9372.

45 J. P. Gann and M. D. Yan, Langmuir, 2008, 24, 5319.

46 X. J. Huang, D. Guduru, Z. K. Xu, J. Vienken and T. Groth, Acta Biomater., 2010, 6, 1099.

$47 \mathrm{H}$. Chen, Y. Chen, H. Sheardown and M. A. Brook, Biomaterials, 2005, 26, 7418.

48 Z. Yang, J. Wang, R. Luo, M. F. Maitz, F. Jing, H. Sun and N. Huang, Biomaterials, 2010, 31, 2072.

49 G. C. M. Steffens, C. Yao, P. Prevel, M. Markowicz, P. Schenck, E. M. Noah and N. Pallua, Tissue Eng., 2004, 10, 1502.

50 S. Van Vlierberghe, M. Sirova, P. Rossmann, H. Thielecke, V. Boterberg, B. Rihova, E. Schacht and P. Dubruel, Biomacromolecules, 2010, 11, 2731.

51 O. Norberg, L. Deng, T. Aastrup, M. D. Yan and O. Ramström, Anal. Chem., 2011, 83, 1000.

52 O. Norberg, L. Deng, M. D. Yan and O. Ramström, Bioconjugate Chem., 2009, 20, 2364-2370.

53 E. P. Kyba and R. A. Abramovitch, J. Am. Chem. Soc., 1980, 102, 735-740.

54 J. P. Collman, N. K. Devaraj, T. P. A. Eberspacher and C. E. D. Chidsey, Langmuir, 2006, 22, 2457.

55 G. C. Tron, T. Pirali, R. A. Billington, P. L. Canonico, G. Sorba and A. A. Genazzani, Med. Res. Rev., 2008, 28, 278.

56 J. C. Jewett and C. R. Bertozzi, Chem. Soc. Rev., 2010, 39, 1272. 57 W. H. Binder and R. Sachsenhofer, Macromol. Rapid Commun., 2007, 28, 15.

58 V. Hong, N. F. Steinmetz, M. Manchester and M. G. Finn, Bioconjugate Chem., 2010, 21, 1912.

59 J. A. Codelli, J. M. Baskin, N. J. Agard and C. R. Bertozzi, J. Am. Chem. Soc., 2008, 130, 11486. 
60 E. M. Sletten and C. R. Bertozzi, Angew. Chem., Int. Ed., 2009, 48, 6974.

61 R. Périona, V. Ferrièresa, M. I. García-Morenob, C. O. Melletb, R. Duvalc, J. M. García Fernándezd and D. Plusquelleca, Tetrahedron, 2005, 61, 9118.

62 R. K. Smith, S. M. Reed, P. A. Lewis, J. D. Monnell, R. S. Clegg, K. F. Kelly, L. A. Bumm, J. E. Hutchison and P. S. Weiss, J. Phys. Chem. B, 2001, 105, 1119.
63 H. C. Kolb, M. G. Finn and K. B. Sharpless, Angew. Chem., Int. Ed., 2001, 40, 2004.

64 D. H. Ess, G. O. Jones and K. N. Houk, Org. Lett., 2008, 10, 1633.

65 B.-B. Ni, C. Wang, H. Wu, J. Pei and Y. Ma, Chem. Commun., 2010, 46, 782.

66 M. Zhang, Z. Liu, W. Tian, D. Liu and X. Ge, Chin. J. Chem., 2011, 69, 1509-1516. 